\title{
Numerical studies of sessile droplet shape with moving contact lines
}

\author{
Jing Liu, Nam-Trung Nguyen, and Yit Fatt Yap \\ School of Mechanical and Aerospace Engineering, Nanyang Technological University, 50 \\ Nanyang Avenue, Singapore, Singapore 639798
}

This paper numerically studies the equilibrium shape of a sessile droplet with moving contact lines. The Navier-Stokes equation was solved through the finite volume method on a Cartesian staggered grid. The level set method was used to track free surface of the immiscible phases. The Navier boundary condition is enforced on the entire solid surface away from the triple contact line to remove the force singularity. The continuum model formulated by Ren and E was used near the contact line ${ }^{1}$. Our code was validated by comparing with other numerical results, and provided a lower mass loss of less than $2 \%$. The method can easily be extended to a three dimensional model. Droplet spreading and recoiling were calculated and discussed with the presented numerical methods. Both two-dimensional and three-dimensional simulation results agree well with experimental observations. 


\section{INTRODUCTION}

A liquid drop deposited on a solid surface forms an air-liquid-solid contact line along the three-phase intersection. The angle between the liquid surface and the solid is called the contact angle. Recently, sessile liquid droplets attract renewed attention from the research community because of the various applications such as assessing wettability ${ }^{2}$ of a solid surface, paper production $^{3}$, magnetic printing ${ }^{4}$ and porous coating ${ }^{5}$. In recent years, the manipulation and actuation of a sessile droplet by termperature ${ }^{6}$, magnetic field ${ }^{7}$, electric field ${ }^{8}$, and surface acoustic wave ${ }^{9}$ become hot topics in the field of microfluidics. The classical static equilibrium shape of a sessile drop is described by the Young's equation. However, numerical investigation of dynamic phenomena of a sessile droplet will encounter problems arising from the dynamic contact angle and the moving contact line. Dussan and Davis showed that if the traditional noslip condition is enforced at the moving contact line by coupling of Navier-Stokes equations, the numerical model will result into stress singularity ${ }^{10}$. Furthermore, the dynamic contact angles exhibit a hysteresis. Thus, the contact line speed needs to be prescribed. The categories of addressing these problems were reviewed by Ren and $\mathrm{E}^{1}$.

The numerical methods for solving contact line problems were reviewed by a number of researchers ${ }^{11-14}$. Diffeerent methods such as volume-of-fluid (VOF) method $^{15}$, Lagrangian finiteelement method, level set method ${ }^{11-12, ~ 16-17, ~ p h a s e ~ f i e l d ~ m e t h o d, ~ t h e ~ s o o t h e d ~ p a r t i c l e ~}$ hydrodynamics method $(\mathrm{SPH})^{13}$, and the recently developed Lattice Boltzmann method ${ }^{18}$ were discussed. Besides these methods, Yokoi et al. used coupled level set and volume-of-fluid method (CLSVOF) to numerically investigate a droplet impacting on a solid surface ${ }^{14}$. They used an asymmetric model to describe three-dimensional problems. Sero-Guillaume et al. employed the minimization of the energy to calculate the shape of a sessile drop with and without magnetic field. The contact angle used the Young's boundary condition ${ }^{19}$. The freely available Surface Evolver (SE) software is popularly used by a number of researchers to model the stable shape of the drops. It is a computer program that minimizes the sum of energies of a surface subject to the various constraints defined by user ${ }^{20}$. The iteration in SE is not base on 
time integral of the fluid flow equations but a search toward minimal energies ${ }^{21}$. Thus, it mainly concerned the finial equilibrium drop shape not the transient process.

Among the above mentioned numerical methods, the level set method is a powerful tool to track the complicated deformed interface between two immiscible phases. Under a shear flow, Spelt numerically investigated a droplet rolling on a solid surface by adding the contact line velocity in the redistance function of level-set approach ${ }^{16-17}$. Ding and Spelt presented a good agreement between the level set method and the diffuse interface method to alleviate the stress singularity problem in the drop spreading problems ${ }^{22}$. Choi and Son studied the droplet motion on a channel surface in the fluid flow ${ }^{11}$. The relationship between the droplet detachment and the flow velocities, as well as the influence of wetting property of the channel surface on droplet merging were investigated. Liu et al. studied the droplet impact on the planar and non-planar solid surface in the $2 \mathrm{D}$ model with consideration of the hysteresis ${ }^{12}$. Very recently, Li, et al. used zero level set to represent the boundary of the drop to solve the single-phase moving contact line problem ${ }^{23}$. However, the disadvantage of the level set method is its mass loss, because the sharpness of the interface is destroyed by under-resolved regions during the discretization process. This mass conservation problem can be solved after several decades of development by employing reintialization equation, seeding particle near the interface, or using global mass correction techniques. The numerical methods used in this paper are more accurate in preserving the mass conservation comparing to other works.

In this paper, the level-set method is used to model a sessile droplet with moving contact line on the Cartesian fixed rectangular grid. The zero level set isocontour represents the interface between two immiscible fluid phases. The interfacial tension is calculated using the continuum surface force model (CSF) ${ }^{24}$. No-slip condition is replaced by Navier boundary condition to avoid the stress singularity. The exact velocity of the contact line utilizes a simple and effective method proposed by Ren and $\mathrm{E}^{1}$ using a continuous function across the triple-phase contact line. Only an assumed static contact angle must be given instead of advancing and receding contact angles. Previously published numerical results were used to validate our code. The present multiphase simulation only has an area loss of less than $2 \%$. Furthermore, we extended the 
method to three dimensions. Both two-dimensional (2D) and three-dimensional (3D) results agree well with the experimental observations. The droplet transition from spherical shape to puddle shape was investigated.
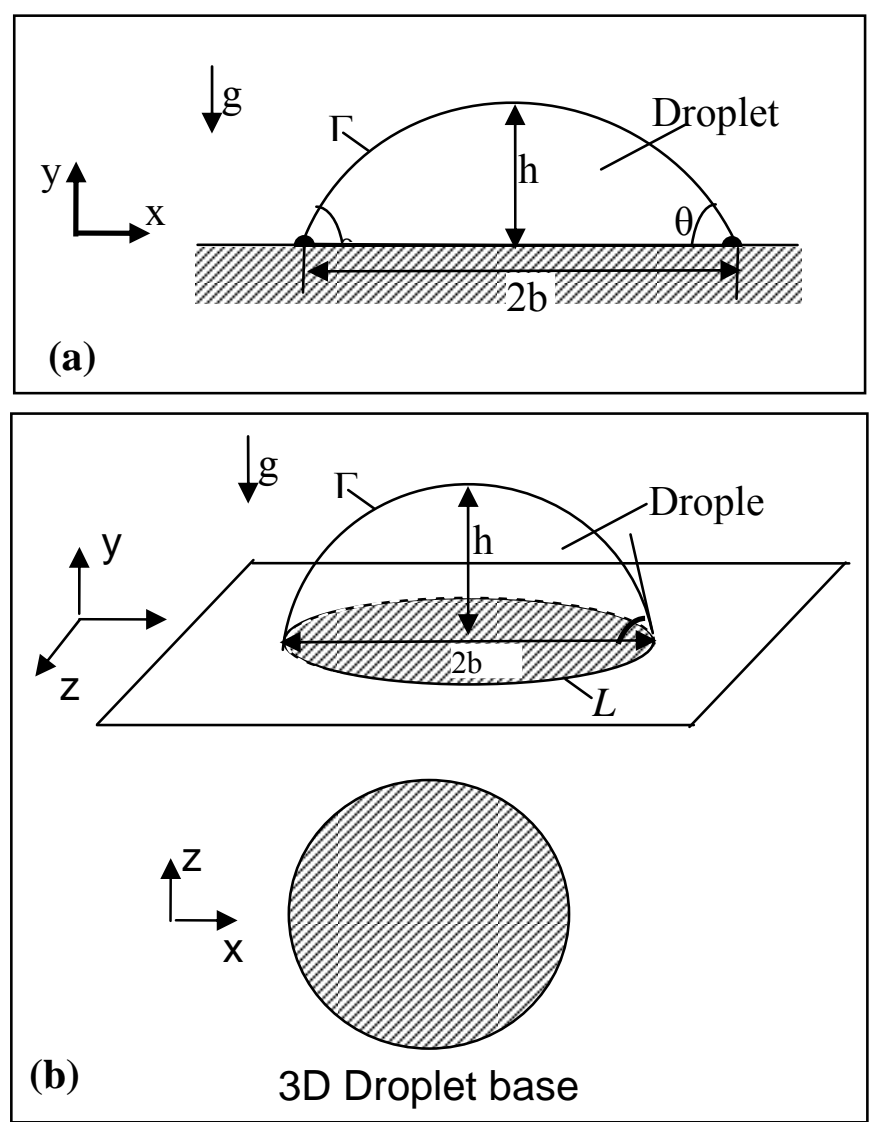

FIG. 1. Schematic of Sessile droplet on the solid surface in the gas with (a) 2D model and (b) 3D model ( $x_{1}$ and $x_{2}$ : contact points of the 2D model, $L$ : contact line of the 3D model, $\theta$ : the contact angel of the three phases, $h$ : droplet height, $2 b$ : droplet base diameter. 


\section{NUMERICAL ANALYSIS}

\section{A. Governing equations}

Figure 1 shows the numerical models investigated in this paper. The free surface $\Gamma$ separates the domain into two regions, i.e. $\Omega_{+}$and $\Omega_{-}$, each filled with the liquid and gas respectively. The droplet sits on the homogeneous surface, and its shape is axial symmetric. Thus, the droplet base is a circle (FIG. 1(b)). The evolution of the surface is captured with the level-set method. The properties across the interface are discontinued. They can be defined using the smoothed Heaviside function $H(\phi)$ by either an arithmetic mean or a harmonic mean. The motions of two immiscible fluids are calculated with two sets of the conservation equations within each region.

The continuity and momentum equations in Cartesian tensor notation for unsteady, viscous, incompressible, immiscible two-phase systems are defined as:

$$
\begin{gathered}
\frac{\partial \rho}{\partial t}+\nabla \bullet(\rho \vec{u})=0 \\
\frac{\partial}{\partial t}(\rho \vec{u})+\nabla \bullet(\rho \vec{u} \vec{u})=-\nabla p+\nabla \bullet\left[\mu\left(\nabla \vec{u}+\nabla \vec{u}^{T}\right)\right]+\vec{F}
\end{gathered}
$$

where $t$ is time, $\vec{u}$ is the velocity vector with three components $\vec{u}=(u, v, w)$ in the $3 \mathrm{D}$ case, $p$ is pressure. The discontinuous phase properties in the whole domain can be defined as an arithmetic mean

$$
\alpha(\phi)=H \alpha_{+}+(1-H) \alpha_{-}
$$

or a harmonic mean

$$
\frac{1}{\alpha(\phi)}=\frac{H}{\alpha_{+}}+\frac{(1-H)}{\alpha_{-}}
$$

The arithmetic mean is used to calculate the density $\rho$, and the harmonic mean is used to calculate the viscosity $\mu$. Since the viscosity is the momentum diffusion coefficient, just as the 
thermal conductivity for heat diffusion, harmonic mean should be accurate for the viscosity. The smoothed Heaviside function $H(\phi)$ is expressed as:

$$
H(\phi)= \begin{cases}0, & \text { if } \phi<-\varepsilon \\ (\phi+\varepsilon) /(2 \varepsilon)+\sin (\pi \phi / \varepsilon) /(2 \pi), & \text { if }|\phi|<\varepsilon \\ 1, & \text { if } \phi>\varepsilon\end{cases}
$$

The parameter $\varepsilon$ is set to 1.5 of the control volume thickness in a uniform grid.

In Eq. (2), $\vec{F}$ can be the interfacial force, magnetic force, and gravity. In our problem, the force component includes both the free surface force $\vec{F}_{\sigma}$ and the gravitational force $\vec{F}_{g}$. The free surface force is determined using the CSF model ${ }^{24}$ :

$$
\vec{F}_{\sigma}=-\sigma \kappa \hat{N}_{F} D\left(\vec{x}-\vec{x}_{f}\right)
$$

where, $\kappa$ is the curvature, $\hat{N}_{F}$ is a normal to the interface, and $D\left(\vec{x}-\vec{x}_{f}\right)$ is the delta function that is zero everywhere except at the interface:

$$
\begin{gathered}
\hat{N}_{F}=\frac{\nabla \phi}{|\nabla \phi|} \\
\kappa=\nabla \bullet \frac{\nabla \phi}{|\nabla \phi|} \\
D\left(\vec{x}-\vec{x}_{f}\right)= \begin{cases}(1+\cos (\pi \phi / \varepsilon)) /(2 \varepsilon), & \text { if }|\phi|<\varepsilon \\
0, & \text { otherwise }\end{cases}
\end{gathered}
$$

The gravity force $\vec{F}_{g}$ is described as

$$
\vec{F}_{g}=f_{g} \hat{g}
$$

where $\hat{g}$ is the unit vector specifying the direction of the body force.

In the work reported here, equation (2) is solved using the finite volume method on Cartesian staggered grid. The velocity-pressure coupling utilizes the SIMPLER algorithm. The combined convection-diffusion effect is predicted by the Power Law. The time integration used a fully implicit scheme. 


\section{B. Free Boundary Solver}

The moving interface $\Gamma$ is traced with the level-set method ${ }^{25}$. The variable $\phi(\vec{x}, t)$ is used to define the interface that separates two regions $\Omega_{+}$and $\Omega_{\text {. }}$ in $R^{3}$. Its value is a signed normal distance function $\phi(\vec{x}, t)$ from the interface:

$$
\phi(\vec{x}, t)=\left\{\begin{array}{c}
-d, \text { if } \vec{x} \in \Omega \\
0, \text { if } \vec{x} \in \Gamma \\
+d, \text { if } \vec{x} \in \Omega_{+}
\end{array}\right.
$$

where the subscripts + and - refer to the liquid phase and the gas phase. The interface $\Gamma$ is represented as the zero level set of function $\phi(\vec{x}, t)$. By taking the time derivative of $\phi(\vec{x}, t)=0$, we get the motion of the interface $\Gamma$ :

$$
\frac{\partial \phi}{\partial t}+\vec{u} \bullet \nabla \phi=0
$$

This function can be solved locally around a narrow band across the interface. Since $\phi$ is the signed normal distance to the interface, which satisfies $|\nabla \phi|=1$.

Numerically, to keep the level set function close to a signed distance function of the interface, a reinitialization procedure is needed to reset the level set function to be a signed distance function of the interface. In the present work, we use the following reinitialization equation for the correction of $\phi$ at time $t^{* 26}$

$$
\begin{gathered}
\frac{\partial \phi^{*}}{\partial t^{*}}=\operatorname{sign}\left(\phi_{0}\right)\left(1-\left|\nabla \phi^{*}\right|\right) \\
\phi_{0}(\vec{x})=\phi(\vec{x}, 0)
\end{gathered}
$$

For numerical purposes it is useful to smooth the signed function as 


$$
\operatorname{sign}\left(\phi_{0}\right)=\phi / \sqrt{\phi^{2}+|\nabla \phi|^{2} \Delta x}
$$

The steady-state solution of the equation will converge to the actual distance, and satisfy $|\nabla \phi|=1$.

Eq. (12) and (13) are special cases of Hamilton-Jacobi equation. They were solved within a band of certain width around the interface instead of over the entire computational domain. This

narrow-band approach is introduced by Peng, et. $\mathrm{al}^{27}$. This approach will not affect the accuracy since the level set value is important only around the interface. This method can reduce one order of magnitude of the computational time. The Hamiltonian of the level set equation and the reinitialization equation are solved by constructing high-order weighted essentially nonoscillatory (WENO) schemes with local Lax-Fridrichs flux ${ }^{28}$ and Godunov flux ${ }^{29}$ respectively. The TVD Runge-Kutta methods were used to integrate the system in time for equation (12) and $(13)^{30}$.

\section{Contact Line Modeling}

The velocity of the contact line was derived by Ren and E using the force balance ${ }^{1}$. The results were utilized later to numerically study the droplet spreading and recoiling by Li et al. ${ }^{23}$ The triple contact line problem was solved based on a single phase, and neglect the influence of surrounding gas on the droplet shape. The method does not consider the contact angle hysteresis. Here, we combined the same continuum model describing the moving contact line of a multiphase system to investigate the drop shape. The effect of surrounding gas is also considered. The details are described in three-dimensional forms as follow.

To remove the force singularity, the slip law is employed by replacing the no-slip condition. That is, along the entire solid surface $y=0$ employed the Navier boundary condition except points near the contact line $\mathrm{e}^{31}$ 


$$
\left\{\begin{array}{l}
u=\lambda \frac{\partial u}{\partial y} \\
v=0 \\
w=\lambda \frac{\partial w}{\partial y}
\end{array}\right.
$$

where, $\lambda$ is the slip length. $\lambda=0$ represents the no-slip condition, and $\lambda=\infty$ is the free-slip condition. In this paper, we assume a partial slip in the vicinity of the solid surface because of the flow friction ${ }^{32}$. In the work of Ren and $E^{1}, \lambda$ is the ratio of viscosity and friction coefficient. In the equation, the velocities parallel to the solid surface $u$ and $w$ are linearly proportional to the local velocity gradient. The velocity of normal to the solid surface $v=0$ represents the no penetration boundary condition.

As long as the slip law is used to avoid force singularity, the speed of the contact must be given to determine the Young's stress and the normal stress of free surface ${ }^{1}$. A simple dynamic velocity was imposed on the points within one grid from the contact line,

$$
\left\{\begin{array}{l}
u=\frac{\sigma}{\alpha}\left(\cos \theta^{*}-\cos \theta\right) \hat{n}_{x} e^{-d_{c}{ }^{2} / \Delta x^{2}} \\
v=0 \\
w=\frac{\sigma}{\alpha}\left(\cos \theta^{*}-\cos \theta\right) \hat{n}_{z} e^{-d_{c}^{2} / \Delta z^{2}}
\end{array}\right.
$$

where $\sigma$ is the surface tension, and $\alpha$ is the effective friction coefficient. $d_{c}$ is the distance of the points to the contact line at the surface of $y=0 . \hat{n}_{x}$ is the component of the normal to the contact line in $x$ direction, and $\hat{n}_{z}$ is the component of the normal to the contact line in the $z$ direction; $\theta^{*}$ is the equilibrium static contact angle; $\theta$ is dynamic contact angle (Fig.1), which is determined by the relationship:

$$
\cos \theta=-\hat{n}_{y} \bullet \nabla \phi
$$

where $\hat{n}_{y}$ is the unit normal vector of the solid surface. This method prescribes the dynamic contact line in a simple way. This method is effective to implement numerically. Since contact 
angle hysteresis is neglected, the advancing and receding contact angles do not need to be predescribed. The condition $|\nabla \phi|=1$ is imposed on the solid boundary. The typical contour of the level set values near the interface is shown in Fig. 2.

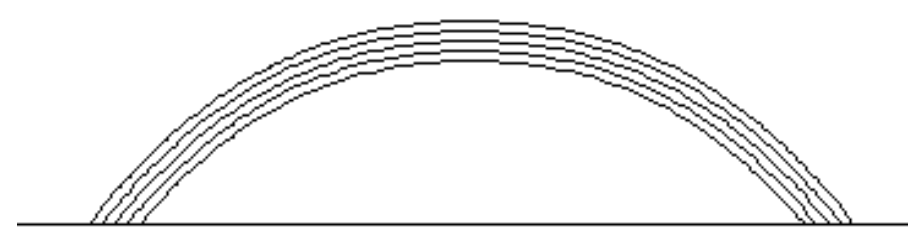

FIG. 2. The level set contour around the free surface in 2D model.

\section{VALIDATIONS AND CASE STUDIES}

The method described above was first validated using a 2D model, FIG. 1(a). A numerical resolution study was carried out based on the example presented by Li et al. ${ }^{23}$. The same parameters and properties are used to compare with the results of Li et al. The initial drop shape is a half circle with a radius of $r=0.4 \mathrm{~m}$ above the $x$-axis. Thus, the initial contact angle is $90^{\circ}$. The static contact angle $\theta^{*}=\pi / 4$. The level set function is positive inside the droplet and negative outside the droplet. The density, the viscosity, the surface tension, the ratio and the slip length are set as $\rho^{+}=1 \mathrm{~kg} / \mathrm{m}^{3} \mu^{+}=2 \mathrm{Pas}, \sigma=0.5 \mathrm{~N} / \mathrm{m}, \frac{\sigma}{\alpha}=1 \mathrm{~m} / \mathrm{s}$, and $\lambda=1 \mathrm{~m}$, respectively. The gravity was taken as $g=9.8 \mathrm{~m}^{2} / \mathrm{s}$. The above parameters are the same used by $\mathrm{Li}$ et al ${ }^{23}$. The density of gas is set to $\rho^{-}=0.001 \mathrm{~kg} / \mathrm{m}^{3}$ to maintain the real density ratio between liquid and gas. The viscosity of gas is assumed to be $\mu^{-}=2$, which is the same as the liquid viscosity. The velocity field will relax to zero when the droplet reaches the equilibrium steady state. Thus, the droplet equilibrium shape is mainly determined by the surface tension, the density of the liquid, and the static contact angle. So the assumed values of viscosity have no significant influence on the shape of the droplet in steady state. The grid independence study is shown in FIG. 3. The dashed line is the result with a mesh of $256 \times 102$ and a time step size $\Delta t=1.25 \times 10^{-3} \mathrm{~s}$ throughout the entire computational domain. The solid line used a mesh of $128 \times 51$ and $\Delta t=2.5 \times 10^{-3} \mathrm{~s}$. The two numerical results are nearly identical. The coarse mesh results into 
more area loss than the fine mesh, and is less than $2 \%$ (FIG. 4). Therefore, adequate numerical accuracy is reached with a mesh $128 \times 51, \Delta t=2.5 \times 10^{-3} \mathrm{~s}$. There are 52 cells across the droplet width.

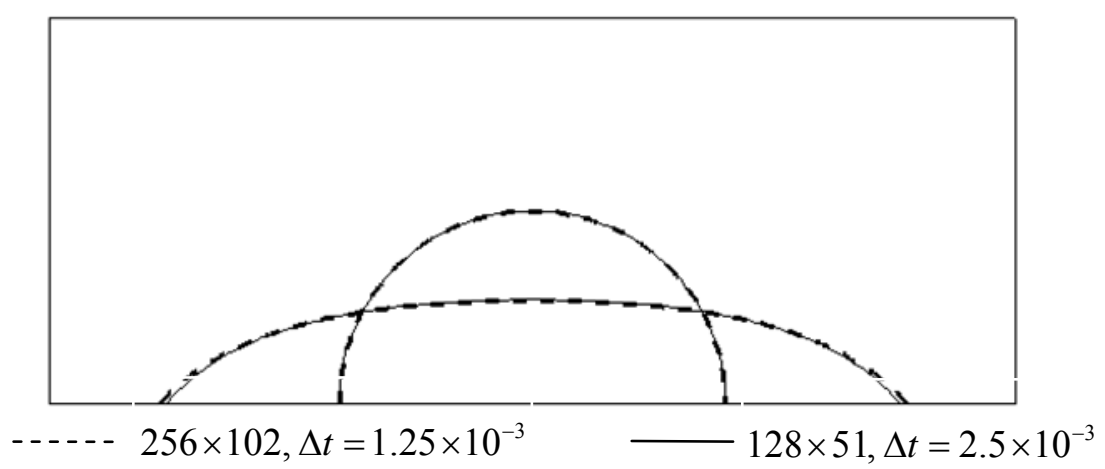

FIG. 3. Grid independence study.

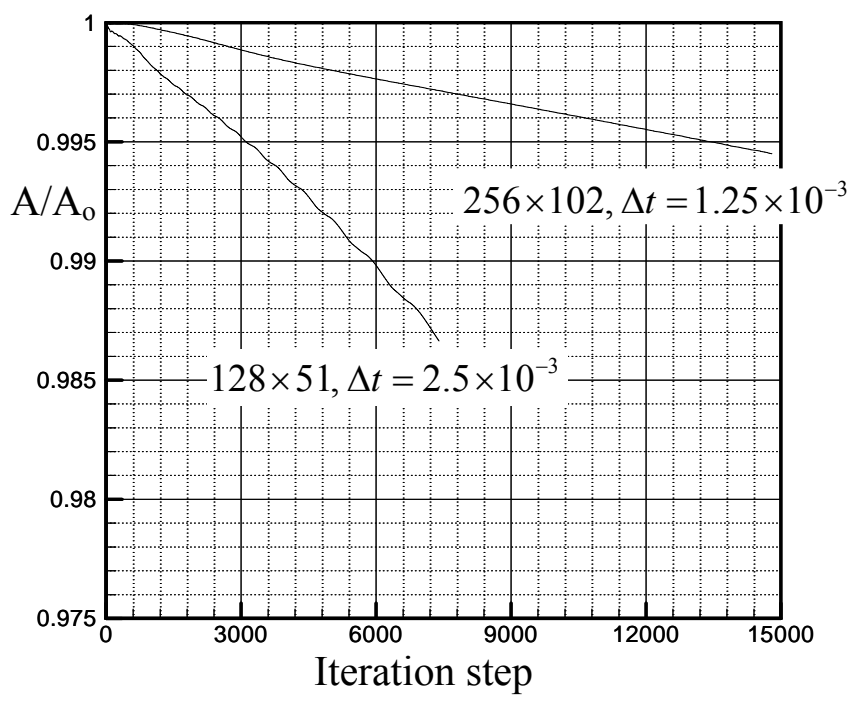

FIG. 4. The normalized area versus iteration step

The present numerical method was first validated with the problem of droplet spreading on the solid surface. Figure 5 compares the shape resulting from our analysis with that of $\mathrm{Li}$ et $\mathrm{al}^{23}$. The results match well in the case of without gravity. Discrepancy occurs if gravity is considered. Because the results proposed by Li et al. seems not preserve the area conservation. The area loss 
is calculated and presented in TABLE I. Their area loss is as much as $16.76 \%$, and the loss of our present method is less than $2 \%$. The area loss versus the iteration step of present method is shown in FIG. 6. The droplet is easy to reach steady state with gravity, therefore, more iteration steps are needed in the absence of the gravity with the same time $\operatorname{step} \Delta t=2.5 \times 10^{-3} \mathrm{~s}$. We put the evolution of droplet profiles from initial shape to the equilibrium shape in FIG. 7.

TABLE I. 2D drop area and area loss of simulation results.

\begin{tabular}{lllll}
\hline \hline & Area (No g) & Area loss(no g) & Area (With g) & Area loss(with g) \\
\hline Li, et al. & 0.251 & $0.039 \%$ & 0.209 & $16.76 \%$ \\
Present method & 0.248 & $1.3 \%$ & 0.246 & $1.9 \%$ \\
\hline \hline
\end{tabular}

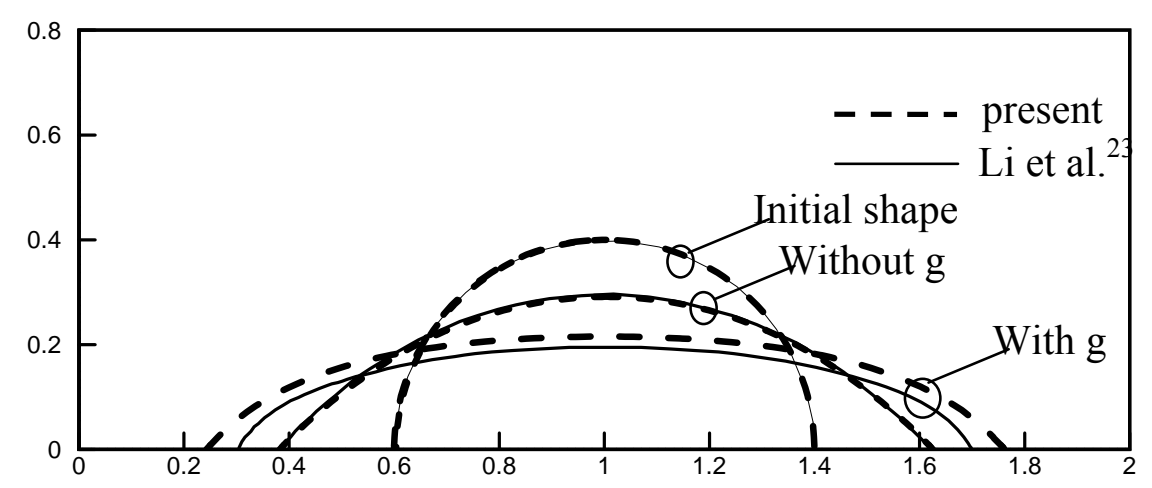

FIG. 5. Droplet spreading with gravity and without gravity. The present results were compared with $\mathrm{Li}$ et $\mathrm{al}^{23}$. 


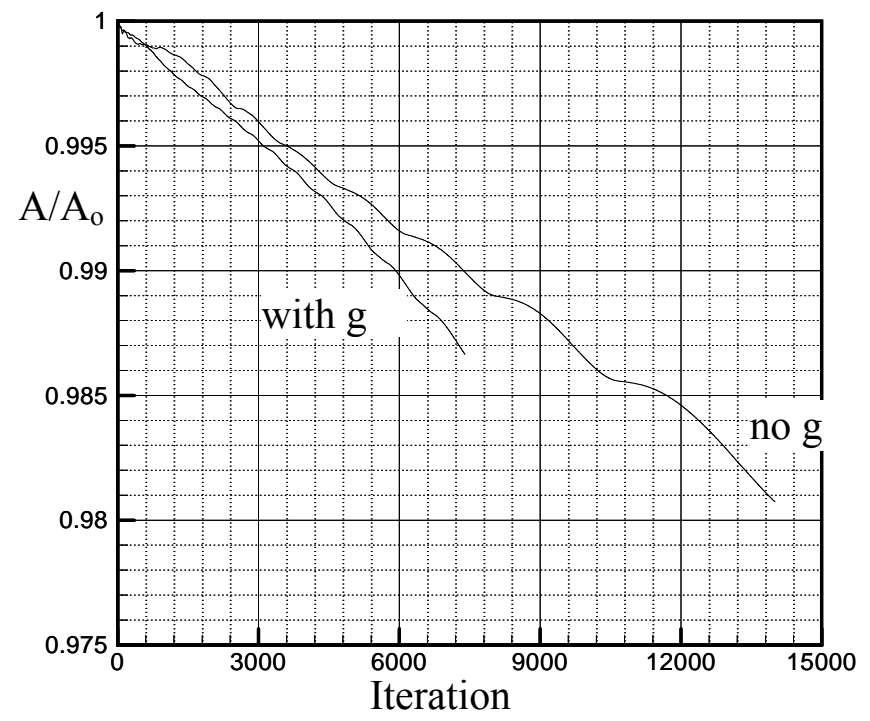

FIG. 6. The comparison of area loss between considering and neglecting gravity $g$.
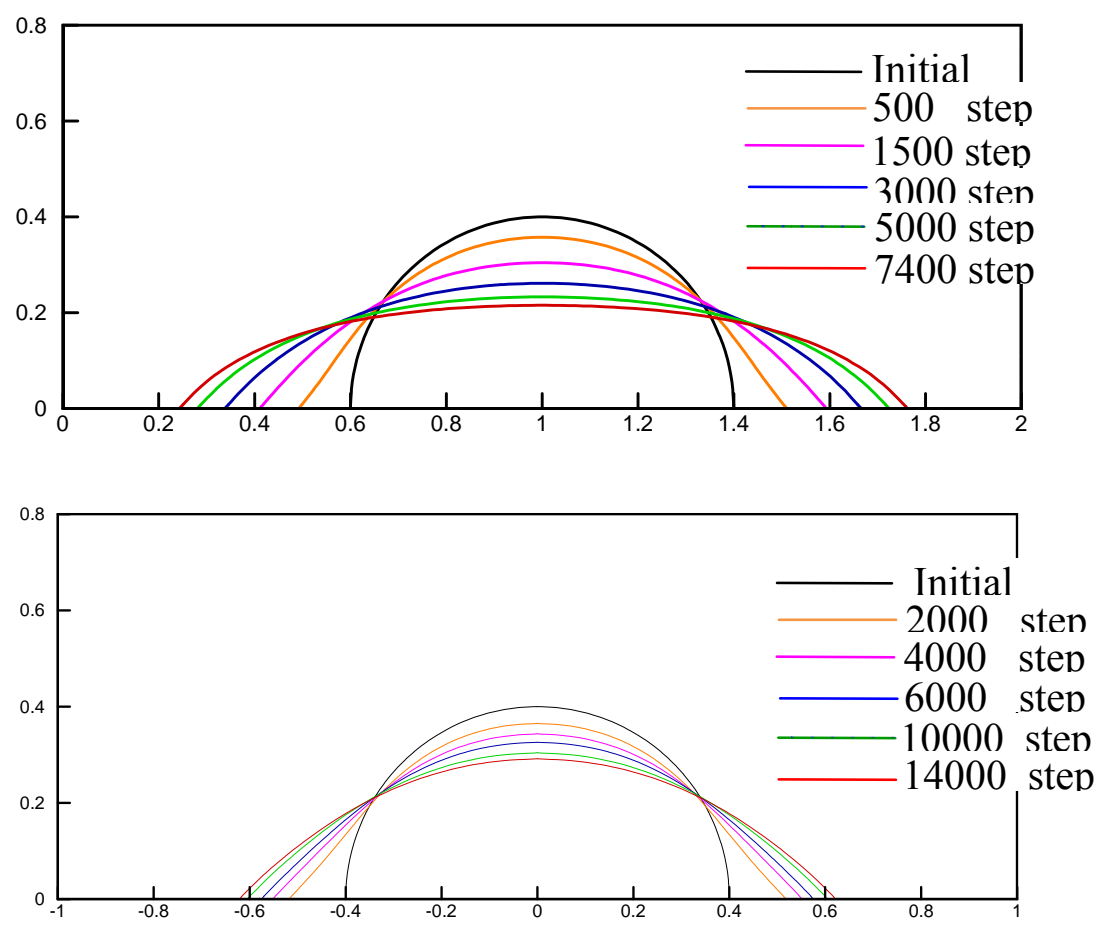

FIG. 7. The changes of the droplet profiles with different iteration step with gravity (a) and without gravity (b). 
Another validation case is droplet recoiling with the gravity effect. Only the static contact angle is changed to $\theta^{*}=3 \pi / 4$, the other parameters are the same as in the last case. The initial droplet shape is still half circle. The comparison results are shown in FIG. 8. They matched well except the area of present result is larger than the results proposed by Li et $\mathrm{al}^{23}$.

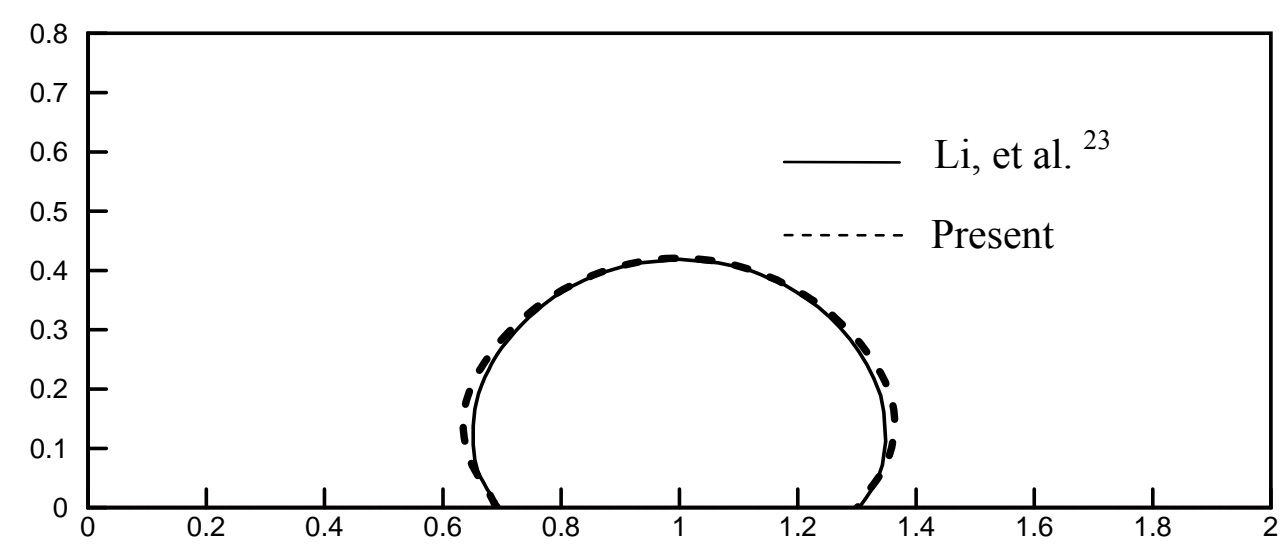

FIG. 8. Droplet recoiling

These two cases demonstrate that the wetting/nonwetting characteristics of the surface determine the spreading or recoiling behavior of the droplet for the same droplet size. Next, we will employ our numerical method to investigate droplet size effect on their corresponding behavior on the same solid surface. The results are compared with experimental data of Extrand and Moon's work ${ }^{2}$ that investigated the shape of three different drop sizes deposited on a variety of polymer and silicon surfaces.

In the experiment of Extrand and Moon ${ }^{2}$, three deionized (DI) water drops were deposited on the solid surface. The volumes were $V=1 \mu \mathrm{L}, 50 \mu \mathrm{L}, 2000 \mu \mathrm{L}$ respectively. If we assume the initial drops are half sphere, the radii are summarized in 
TABLE II. The solid surface is hydrophobic, and the static contact angle is $\theta^{*}=108^{\circ}$. The properties of the liquids used in the simulation are, the density $\rho^{+}=998 \mathrm{~kg} / \mathrm{m}^{3}, \rho^{-}=1.25 \mathrm{~kg} / \mathrm{m}^{3}$, the viscosity $\mu^{+}=\mu^{-}=2 \mathrm{Pas}$, the surface tension $\sigma=0.072 \mathrm{~N} / \mathrm{m}$, the ratio $\frac{\sigma}{\alpha}=1 \mathrm{~m} / \mathrm{s}$, and the slip length is one grid distance $\lambda=\Delta x^{12,17}$. As an input parameter, Spelt discussed its value influence on the contact line region ${ }^{16}$. As mentioned above, the assumed values of the viscosity have no effect on the equilibrium steady-state shape, FIG. 9(a). However, it still plays an important role in the transient process before the velocity relaxes to zero i.e. before the droplet reaches its steady state. The droplet was first studied based on a 2D model. The initial drop is a semi circle sitting on the solid surface. Given the symmetry of the problem, only half of the domain is simulated. For the small drop $V=1 \mu 1$, recoiling will happen as shown in FIG. 9(a). Increasing the drop size to $V=50 \mu \mathrm{l}$, its shape begins to spread to a puddle form instead of remain the spherical cap, FIG. 9(b). If the drop volume is sufficiently large, it will assume the puddle form under the influence of gravity, FIG. 9(c). This phenomenon can be explained with a dimensionless number, the Bond number $B o=\frac{\rho g R^{2}}{\sigma}$, where $R$ is of the order of the drop radius. If $B o<1$, the drop remains approximately spherical, and it displays recoiling. Otherwise, the gravity flattens the drop on the solid surface, and it spreads to a puddle form. The values of Bo of three drops are shown in Table II. The size of the second drop is the transient point for drop beginning to spreading. The capillary length of the system $l=\sqrt{\frac{\sigma}{\rho g}}$ is $2.7 \mathrm{~mm}$. If the drop radius is less than this value, the drop assumes a spherical shape. If the radius is larger than the capillary length, the drop assumes a puddle shape. These drop behavior is consistent with the experiment results reported by Extrand and Moon ${ }^{2}$ which indicate hat the volume transition point was $V=39 \mu \mathrm{L}$, and the spreading phenomena occurred with a base diameter of $2 b=4.3 \mathrm{~mm}$ and a height of $h=2.7 \mathrm{~mm}$.

If the problem is solved in a $2 \mathrm{D}$ Cartesian coordinate, the numerical results can not be validated, because the 2D drops have a cylindrical shape instead of the actual 3D spherical shape. 
Thus, the 2D model is not axial symmetric. To describe the problems more accurately, three dimensional models were built. The initial drop shape is a half sphere. The radii and the liquid properties are the same as those of the 2D models. One quarter of drop was simulated according its symmetry. The numerical results are shown in FIG. 10 . They show a go Pa s.eement with the experiment observation ${ }^{2}$. Table III compares the drop height and the base diameter resulting from numerical simulation and experiments.

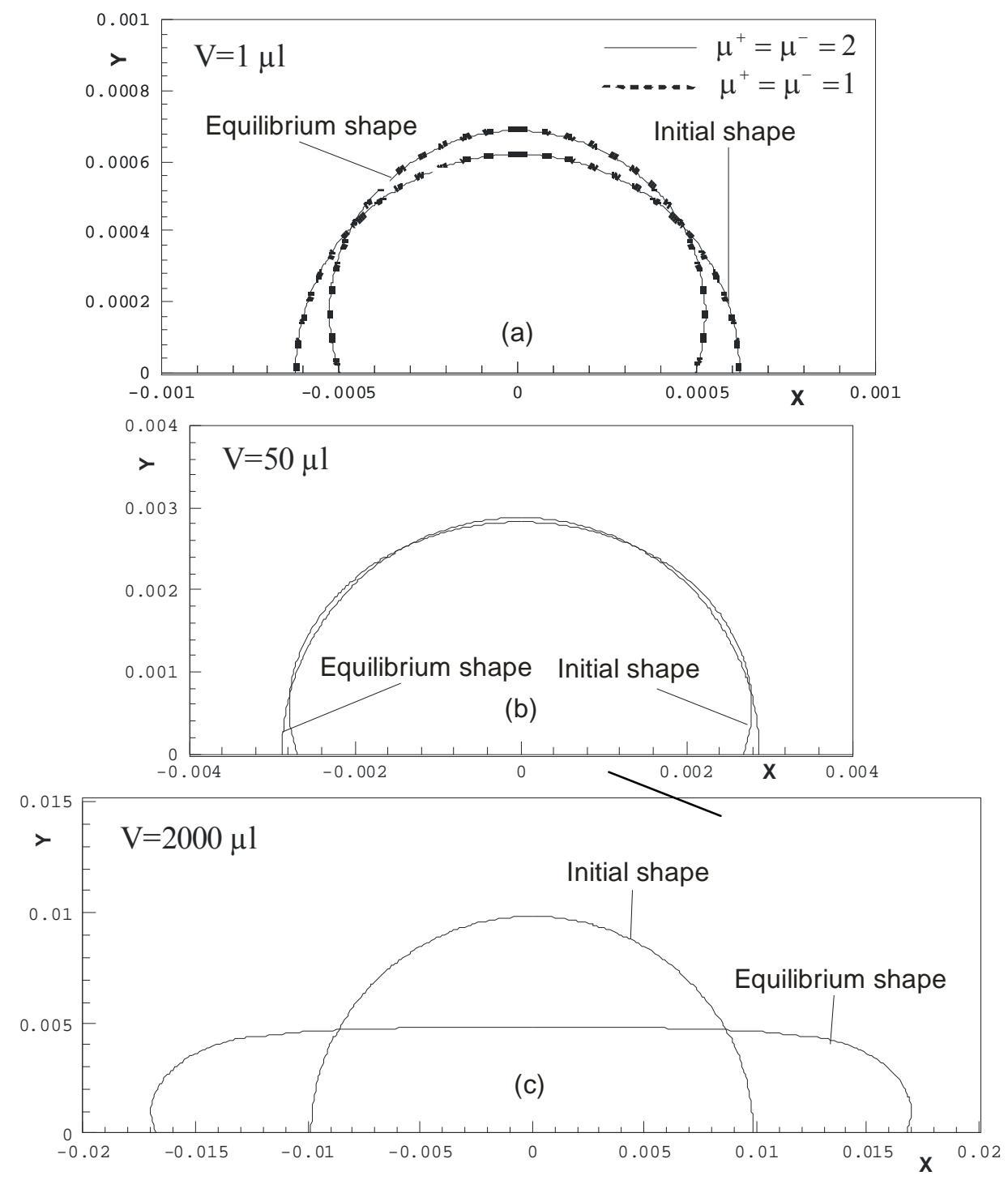

FIG. 9. Equilibrium shape of droplets with initial radii of (a) $r=0.62 \mathrm{~mm}$, (b) $r=2.88 \mathrm{~mm}$ and (c) $r=9.85 \mathrm{~mm}$. 
TABLE II. The drops volume, radii, and Bond number Bo.

\begin{tabular}{cccc}
\hline \hline & Volume $(\mu 1)$ & Radii $(\mathrm{mm})$ & Bo \\
\hline Drop 1 & 1 & 0.62 & 0.052 \\
Drop 2 & 50 & 2.88 & 1.127 \\
Drop 3 & 2000 & 9.85 & 13.18 \\
\hline \hline
\end{tabular}

(a)

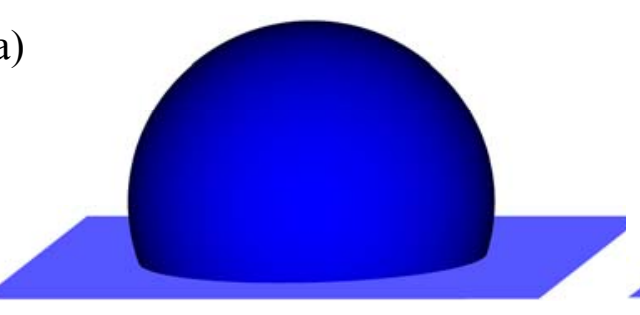

(b)

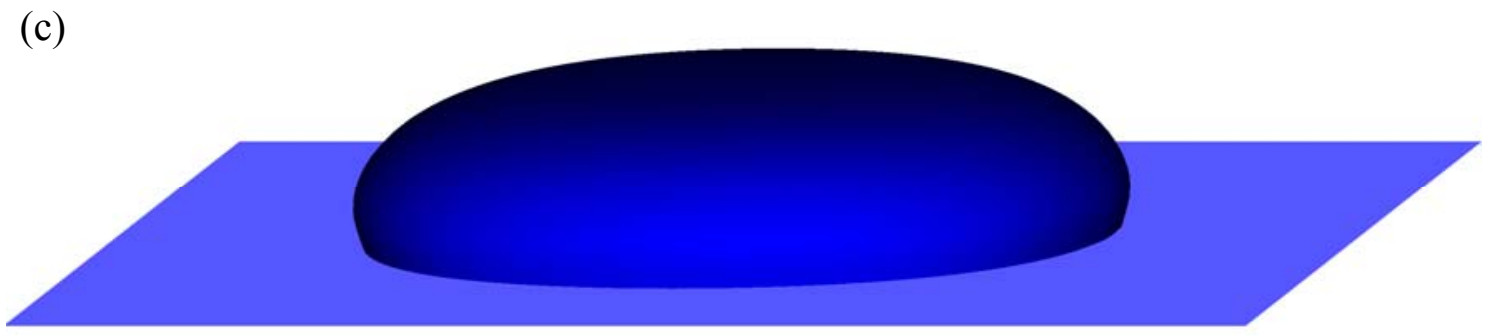

FIG. 10. Drop shapes of 3D numerical results $V=1 \mu \mathrm{L}$ (a), $V=50 \mu \mathrm{L}$ (b) $V=2000 \mu \mathrm{L}$.

TABLE III. Drop height $(h)$ and base diameter $(2 b)$ for sessile drop sitting on the solid surface.

\begin{tabular}{lllllll}
\hline \hline & \multicolumn{2}{c}{ Present (2D) } & \multicolumn{2}{c}{ Present (3D) } & \multicolumn{2}{c}{ Extrand and Moon $^{2}$} \\
& $2 b(\mathrm{~mm})$ & $h(\mathrm{~mm})$ & $2 b(\mathrm{~mm})$ & $h(\mathrm{~mm})$ & $2 b(\mathrm{~mm})$ & $h(\mathrm{~mm})$ \\
\hline Drop1 & 1.02 & 0.69 & 1.02 & 0.72 & $1.052 \pm 0.002$ & $0.82 \pm 0.02$ \\
Drop2 & 5.54 & 2.82 & 5.26 & 2.9 & $5.46 \pm 0.1$ & $2.91 \pm 0.04$ \\
Drop3 & 33.6 & 4.85 & 23.6 & 5.1 & $26.52 \pm 1.35$ & $4.56 \pm 0.05$ \\
\hline \hline
\end{tabular}

\section{CONCLUSIONS}

The equilibrium shape of a sessile droplet was investigated numerically with moving contact line. The continuum model proposed by Ren and E proved to be suitable to describe the 
dynamics of the spreading and recoiling of the droplet on the solid surface in the present numerical method. The simulation was performed by considering both gas and liquid phases. The steady state of the droplet was reached by using the sliding velocity of the contact line. The deformed drop shape was determined by the level set method. The discontinuous properties of the whole domain were defined with soothed Heaviside function. The equilibrium steady-state shape is mainly determined by the drop size, surface tension, gravity, and poor or good wettability performance of the solid surface. For the fixed droplet size, the droplet can display spreading or recoiling depending on the wetting/nonwetting characteristics of the solid surface. On the non-wetting surface of the solid by the liquid, the drop shape results from the balance of the surface tension and the gravity. The sufficiently small volume drop assumes a spherical shape because the surface tension dominates, while the shape of a larger drop is controlled by both gravitational force and surface tension.

${ }^{1}$ W. Ren and W. E, "Boundary conditions for the moving contact line problem",Phys. Fluids 19 (2) (2007).

${ }^{2}$ C. W. Extrand and S. I. Moon, "When Sessile Drops Are No Longer Small: Transitions from Spherical to Fully Flattened",Langmuir 26 (14), 11815-11822 (2010).

${ }^{3} \mathrm{C}$. Aydemir, "Time-dependent behavior of a sessile water droplet on various papers",Int. J. Polym. Mater. 59 (6), 387-397 (2010).

${ }^{4}$ C. Zeller and W. W. Chen, "The instability of a sessile drop of magnetic fluid",J. Magn. Magn. Mater. 39 (1-2), 165-168 (1983).

${ }^{5}$ B. Drevet, O. Pajani and N. Eustathopoulos, "Wetting, infiltration and sticking phenomena in Si3N4 releasing coatings in the growth of photovoltaic silicon",Sol. Energy Mater. Sol. Cells 94 (3), 425-431 (2010).

${ }^{6}$ H. B. Nguyen and J. C. Chen, "A numerical study of thermocapillary migration of a small liquid droplet on a horizontal solid surface",Phys. Fluids 22 (6), 1-12 (2010).

${ }^{7}$ N.-T. Nguyen, G. Zhu, Y.-C. Chua, V.-N. Phan and S.-H. Tan, "Magnetowetting and Sliding Motion of a Sessile Ferrofluid Droplet in the Presence of a Permanent Magnet",Langmuir 26 (15), 12553-12559 (2010).

${ }^{8}$ S. Haeberle and R. Zengerle, "Microfluidic platforms for lab-on-a-chip applications",Lab Chip 7 (9), 1094-1110 (2007).

${ }^{9}$ L. Y. Yeo and J. R. Friend, "Ultrafast microfluidics using surface acoustic waves",Biomicrofluidics 3 (1) (2009).

${ }^{10}$ E. B. Dussan V and S. H. Davis, "ON THE MOTION OF A FLUID-FLUID INTERFACE ALONG A SOLID SURFACE",J. Fluid Mech. 65 (Part 1), 71-95 (1974).

${ }^{11}$ J. Choi and G. Son, "Numerical study of droplet motion in a microchannel with different contact angles",J. Mech. Sci. Technol. 22 (12), 2590-2599 (2008). 
${ }^{12}$ H. Liu, S. Krishnan, S. Marella and H. S. Udaykumar, "Sharp interface Cartesian grid method II: A technique for simulating droplet interactions with surfaces of arbitrary shape",J. Comput. Phys. 210 (1), 32-54 (2005).

${ }^{13}$ A. K. Das and P. K. Das, "Equilibrium shape and contact angle of sessile drops of different volumesComputation by SPH and its further improvement by DI",Chem. Eng. Sci. 65 (13), 4027-4037 (2010).

${ }^{14}$ K. Yokoi, D. Vadillo, J. Hinch and I. Hutchings, "Numerical studies of the influence of the dynamic contact angle on a droplet impacting on a dry surface",Phys. Fluids 21 (7) (2009).

${ }^{15}$ M. Renardy, Y. Renardy and J. Li, "Numerical Simulation of Moving Contact Line Problems Using a Volume-of-Fluid Method",J. Comput. Phys. 171 (1), 243-263 (2001).

${ }^{16} \mathrm{P}$. D. M. Spelt, "A level-set approach for simulations of flows with multiple moving contact lines with hysteresis",J. Comput. Phys. 207 (2), 389-404 (2005).

${ }^{17}$ P. D. M. Spelt, "Shear flow past two-dimensional droplets pinned or moving on an adhering channel wall at moderate Reynolds numbers: A numerical study",J. Fluid Mech. 561, 439-463 (2006).

${ }^{18}$ X. Q. Xing, D. L. Butler and C. Yang, "Lattice Boltzmann-based single-phase method for free surface tracking of droplet motions",Int. J. Numer. Methods Fluids 53 (2), 333-351 (2007).

${ }^{19}$ O. E. Sero-Guillaume, D. Zouaoui, D. Bernardin and J. P. Brancher, "The shape of a magnetic liquid drop",J. Fluid Mech. 241, 215-232 (1992).

${ }^{20}$ K. Brakke, "The Surface Evolver",Experimental Mathematics 1 (2), 141-165 (1992).

${ }^{21}$ S. H. Collicott and M. M. Weislogel, "Computing Existence and Stability of Capillary Surfaces Using Surface Evolver",AIAA Journal 42 (2), 289-295 (2004).

${ }^{22}$ H. Ding and P. D. M. Spelt, "Inertial effects in droplet spreading: A comparison between diffuseinterface and level-set simulations",J. Fluid Mech. 576, 287-296 (2007).

${ }^{23}$ Z. Li, M. C. Lai, G. He and H. Zhao, "An augmented method for free boundary problems with moving contact lines",Comput. Fluids 39 (6), 1033-1040 (2010).

${ }^{24}$ J. U. Brackbill, "A continuum method for modeling surface tension",J. Comput. Phys. 100 (2), 335-354 (1992).

${ }^{25}$ S. Osher and J. A. Sethian, "Fronts propagating with curvature-dependent speed: Algorithms based on Hamilton-Jacobi formulations",J. Comput. Phys. 79 (1), 12-49 (1988).

${ }^{26}$ M. Sussman, "A level set approach for computing solutions to incompressible two-phase flow",J. Comput. Phys. 114 (1), 146-159 (1994).

${ }^{27}$ D. Peng, B. Merriman, S. Osher, H. Zhao and M. Kang, "A PDE-Based Fast Local Level Set Method",J. Comput. Phys. 155 (2), 410-438 (1999).

${ }^{28} \mathrm{C}$. W. Shu and S. Oshert, "Efficient implementation of essentially non-oscillatory shock-capturing schemes, II",J. Comput. Phys. 83 (1), 32-78 (1989).

${ }^{29}$ G. S. Jiang and D. Peng, "Weighted ENO schemes for Hamilton-Jacobi equations",SIAM J. Sci. Comput. 21 (6), 2126-2143 (2000).

${ }^{30}$ G. S. Jiang and C. W. Shu, "Efficient implementation of weighted ENO schemes",J. Comput. Phys. 126 (1), 202-228 (1996).

${ }^{31}$ G. K. Batchelor, H. K. Moffatt and M. G. Worster, Perspectives in fluid dynamics. (cambridge University Press, Cambridge, 2000).

${ }^{32} \mathrm{~S}$. Ganesan and L. Tobiska, "Modelling and simulation of moving contact line problems with wetting effects",Comput Visual Sci 12 (7), 329-336 (2009). 